\title{
PROGRESS IN MIXED EULERIAN-LAGRANGIAN FINITE ELEMENT SIMULATION OF FORMING PROCESSES
}

\author{
J. HUÉTINK AND P. T, VREEDE
}

University of Twente, Department of Mechanical Engineering, P.O. Box 217, 7500 AE Enschede, The Netherlands

J. VAN DER LUGT

Hoogovens Steelworks, Department of Process Technology of Rolling Mills, P.O. Box 10000, 1970 CA Ijmuiden, The Netherlands

\begin{abstract}
SUMMARY
A review is given of a mixed Eulerian-Lagrangian finite element method for simulation of forming processes. This method permits incremental adaptation of nodal point locations independently from the actual material displacements. Hence numerical difficulties due to large element distortions, as may occur when the updated Lagrange method is applied, can be avoided. Movement of (free) surfaces can be taken into account by adapting nodal surface points in a way that they remain on the surface. Hardening and other deformation path dependent properties are determined by incremental treatment of convective terms. A local and a weighed global smoothing procedure is introduced in order to avoid numerical instabilities and numerical diffusion. Prediction of contact phenomena such as gap opening and/or closing and sliding with friction is accomplished by a special contact element. The method is demonstrated by simulations of an upsetting process and a wire drawing process.
\end{abstract}

\section{INTRODUCTION}

Problems of fluid dynamics are commonly formulated in a spatial Eulerian reference system. In numerical simulations of fluid problems an Eulerian formulation with a spatially fixed grid has some disadvantages when free surfaces or fluid structure interaction with large displacements have to be taken into account. To solve these kinds of problems, Hughes et al. ${ }^{12}$ and Donea ${ }^{3}$ introduced the Arbitrary Eulerian-Lagrangian description in fluid dynamics.

In simulations of forming processes by the finite element method the (updated) Lagrangian formulation is commonly applied. An advantage of a Lagrangian description is that deformation history dependent properties (strain hardening, etc.) can easily be taken into account, and the changing shape of the formed product can be followed. The updated Lagrange method is restricted with respect to the deformation range because the element mesh may be completely distorted after a number of steps. Rezoning ${ }^{4}$ (remeshing) is necessary, but is in some cases not efficient. In a (nearly) steady-state problem an Eulerian description with a spatial fixed grid would be more appropriate. In other non-steady-state problems mesh refinement may be desired in an area (with high rate of deformation or with changing contact between tool and nodal material points) independently from the material flow. In these cases very frequent rezoning is required.

To avoid these problems, a procedure has been derived in which the location of the nodal points can be disconnected from the displacement of the material. In this way conditions for free or coupled surface movements can be satisfied. An updated Lagrange approach as well as an 
Eulerian approach can be regarded as special cases of the procedure, therefore it is called the mixed Eulerian-Lagrangian formulation. The first results have been presented at the first NUMIFORM conference. ${ }^{8}$ A similar method is developed by Schreurs and co-workers. ${ }^{20,21}$ The procedure has been developed simultaneously with the Arbitrary Eulerian-Lagrangian formulation for fluid dynamics. However, in Newtonian fluid problems no deformation history dependent material properties have to be taken into account and therefore no particular attention has been paid to this aspect in simulation of fluid problems. In an Eulerian discretization, incremental updating at grid points of history dependent material properties (i.e. strain hardening) cannot easily be carried out by adding a Lagrangian material (strain) increment because grid points do not continuously coincide with the same material particles. In a steady-state solution only, integration along stream lines can be applied. In forming processes history dependent effects can generally not be neglected. Hence in the present formulation much attention is paid to the prediction of history dependent effects.

The initial formulation ${ }^{8}$ for updating the state at integration points appeared to result in numerical instabilities depending on the size of the displacement increments. By applying local and global smoothing introduced by Hinton and Gampbell, ${ }^{6}$ numerical instabilities can be avoided. However, numerical diffusion due to smoothing may occur. This numerical diffusion can be reduced by using a weight factor for the amount of global smoothing. This weight factor depends on the ratio of the displacement increment and the element size and is determined by a number of numerical experiments. ${ }^{9}$

\section{INCREMENTAL FORMULATION}

The material behaviour is assumed to satisfy generally applied constitutive equations for elastic-plastic material including thermal effects. These equations can be written as

$$
\stackrel{\nabla}{\boldsymbol{\sigma}}=\frac{\dot{\rho}}{\rho} \boldsymbol{\sigma}+\mathbb{C}: \boldsymbol{d}+\mathbb{K} \dot{T}-\boldsymbol{\Phi}^{t}
$$

where $\underset{\sigma}{\sigma}$ is the Jaumann rate of the Cauchy stress tensor $\sigma, \rho$ is the mass density, $\mathbb{C}$ is the elastic plastic 'tangent modulus' tensor depending on the material properties, the stress and the deformation history. The tensor $\boldsymbol{d}$ is the rate of deformation tensor. The tensor $\mathbb{K}$ denotes the temperature dependence of the material properties including the thermal expansion. $T$ is the absolute temperature, and the tensor $\boldsymbol{\Phi}^{t}$ represents the dissipation related to the time dependent material behaviour.

The equilibrium conditions yield

$$
\boldsymbol{\sigma} \cdot \bar{\nabla}+\rho \mathbf{f}=\boldsymbol{\phi}
$$

and

$$
\rho c \dot{T}-\vec{\nabla} \cdot(\lambda \cdot \vec{\nabla} T)-q=\phi
$$

where $f$ represents an external body force per unit of mass, $\lambda$ is the thermal conductivity tensor and $q$ is the mechanical energy rate, $q=\sigma: d$.

Non-linearity and path dependence necessitate an incremental solving procedure. The same procedure as for an updated Lagrange method is followed for calculating a material displacement increment $\left(\Delta u^{N}\right)$ at the nodal points. The updated Lagrange method is supposed to be well known, hence no further explanation is given.

Deviation from the updated Lagrange procedure occurs in the way the mesh is updated and a new state (stress, strain, etc.) is calculated after solving the material displacement increment. In 
the mixed Eulerian-Lagrangian formulation material velocities and displacements are uncoupled from nodal (grid) point velocities and displacements. The velocity of a material particle $x^{\mathrm{m}}$ at spatial point $\mathbf{x}$ and time $t$ is defined by

$$
\mathbf{v}^{\mathrm{m}}=\frac{\mathrm{d} \mathbf{x}^{\mathrm{m}}}{\mathrm{d} t}=\dot{\mathbf{x}}^{\mathrm{m}}
$$

The velocity of a grid point $\mathbf{x}^{\mathrm{g}}$ coinciding with $\mathbf{x}$ at time $t$ is represented by

$$
\mathbf{v}^{\mathrm{g}}=\frac{\mathrm{dx}}{\mathrm{d} t}=\dot{\mathbf{x}}^{\mathrm{g}}
$$

In order to update the material associated quantities (stress, strain, etc.) in grid points, we have to consider the rate of change of these quantities in each grid point. The rate of change in a grid point differs from the material rate of change. The updating procedure will be illustrated by considering the effective (von Mises) strain. The material rate of change of the effective strain in a material point $x^{\mathrm{m}}$ is related to the rate of deformation tensor $d$ by

$$
\dot{\varepsilon}^{\mathrm{m}}=\sqrt{\frac{2}{3} \boldsymbol{d}: \boldsymbol{d}}
$$

where the rate of deformation tensor $\boldsymbol{d}$ is defined by

$$
d=\frac{1}{2}\left(\vec{\nabla} \mathbf{v}^{\mathrm{m}}+\mathbf{v}^{\mathrm{m}} \stackrel{\grave{\nabla}}{)}\right)
$$

This strain rate can also be expressed as

$$
\dot{\varepsilon}^{\mathrm{m}}=\left(\frac{\mathrm{d} \varepsilon}{\mathrm{d} t}\right)^{\mathrm{m}}=\left(\frac{\partial \varepsilon}{\partial \mathrm{t}}\right)+\dot{\mathrm{x}}^{\mathrm{m}} \cdot \vec{\nabla} \varepsilon
$$

The rate of change of the effective strain in a grid point $\mathbf{x}^{\mathrm{g}}$ can be expressed as

$$
\dot{\varepsilon}^{\mathrm{g}}=\left(\frac{\mathrm{d} \varepsilon}{\mathrm{d} t}\right)^{\mathrm{g}}=\left(\frac{\partial \varepsilon}{\partial t}\right)+\dot{\mathbf{x}}^{\mathrm{g}} \cdot \vec{\nabla} \varepsilon
$$

Solving the spatial rate of change $\partial \varepsilon / \partial t$ from (8) and substituting into (9) results in

$$
\dot{\varepsilon}^{\mathrm{g}}=\dot{\varepsilon}^{\mathrm{m}}+\left(\dot{\mathbf{x}}^{\mathrm{g}}-\dot{\mathbf{x}}^{\mathrm{m}}\right) \cdot \vec{\nabla} \varepsilon
$$

It is obvious that uncoupling of material and grid point velocities (and displacements) implies that, in addition to the incremental (strain and stress) calculation as in the updated method, convection must be taken into account in order to be able to update the state at the grid points.

The strain in a grid point $\mathbf{x}^{\mathrm{g}}$ after a time increment $\Delta t$ is approximated using an incremental form of $(10)$ by

$$
\varepsilon\left(\mathbf{x}^{\mathrm{g}}+\Delta \mathbf{x}^{\mathrm{g}}, t+\Delta t\right)=\varepsilon\left(\mathbf{x}^{\mathrm{g}}, t\right)+\Delta \varepsilon^{\mathrm{m}}+\left(\Delta \mathbf{x}^{\mathrm{g}}-\Delta \mathbf{x}^{\mathrm{m}}\right) \cdot \vec{\nabla} \varepsilon
$$

The second term on the right hand side of (11) equals the strain increment as in the updated Lagrange method; the third term represents the convective strain increment.

The material displacement increment $\Delta \mathbf{x}^{\mathbf{m}}$ is derived from the solved nodal point material displacement increment $\Delta \mathbf{u}^{N}$

$$
\Delta \mathbf{x}^{\mathrm{m}}=\Delta \mathbf{u}=\hat{\mathbf{v}} \Delta t=\psi^{N} \Delta \mathbf{u}^{N}
$$

The grid displacement increment $\Delta \mathbf{x}^{\mathrm{g}}$ can be determined independently from the material displacement. In the pure Eulerian case $\Delta \mathbf{x}^{\mathrm{g}}$ vanishes, in the updated Lagrange case $\Delta \mathbf{x}^{8}$ equals $\Delta u$. At boundary points the grid point locations must be updated in such a way that they remain 
on the boundary surface, hence the pure Eulerian case is limited to problems with spatially fixed boundaries. The grid displacement increment can be related to nodal grid point displacements similar to equation (12). Algorithms for automatic control of the nodal grid points locations are presented by Schreurs and co-workers ${ }^{20,21}$ and Ponthon. ${ }^{17,18}$

The determination of the convective strain increment complicates the solution procedure considerably because the strain gradient is needed. Strains are generally not continuous at element boundaries, hence the gradient of the strains cannot reliably be determined directly at element level. For example, in elements with linear interpolation of the displacement, the strain gradient vanishes. The convective terms are therefore calculated using the strain in the current element and the adjacent elements. In every node average nodal strains $\varepsilon^{N}$ are calculated from all elements that are connected to a particular nodal point. Then by interpolation of these mean nodal point values a continuous strain field is obtained.

$$
\varepsilon^{*}=\psi^{N} \varepsilon^{N}
$$

Usually these mean nodal values are calculated for post processing purposes. Average nodal stresses calculated in the same way are used for error estimating by Zienkiewicz and Zhu. ${ }^{24}$

This additional continuous strain field serves for the determination of the strain gradient in (11), resulting in

$$
\varepsilon\left(\mathbf{x}^{\mathrm{g}}+\Delta \mathbf{x}^{\mathrm{g}}, t+\Delta t\right)=\varepsilon\left(\mathbf{x}^{\mathrm{g}}, t\right)+\Delta \varepsilon^{\mathrm{m}}+\left(\Delta \mathbf{x}^{\mathrm{g}}-\Delta \mathbf{u}\right) \cdot \overrightarrow{\mathbf{\nabla}} \psi^{N} \varepsilon^{N}
$$

It was observed that this formulation showed numerical instabilities in some cases, depending on the size of the displacement increments. These numerical instabilities can be avoided by applying local and global smoothing. ${ }^{6}$ First local least square smoothing is applied at element level prior to the calculation of mean nodal point values, i.e. in four node elements the mean value of the four integration points is taken. Global smoothing implies that in equation (14) the (known) initial state $\varepsilon\left(\mathbf{x}^{\mathrm{g}}, t\right)$ is replaced by the continuous strain of equation (13).

$$
\varepsilon\left(\mathbf{x}^{\mathrm{g}}+\Delta \mathbf{x}^{\mathrm{g}}, t+\Delta t\right)=\psi^{N}\left(\mathbf{x}^{\mathrm{g}}\right) \varepsilon^{N}(t)+\Delta \varepsilon^{\mathrm{m}}+\left(\Delta \mathbf{x}^{\mathrm{g}}-\Delta \mathbf{u}\right) \cdot \vec{\nabla} \psi^{N} \varepsilon^{N}
$$

However, it was observed that successive local and global smoothing results in numerical diffusion. ${ }^{9}$ Besides, if stresses are smoothed in this way then this stress field will generally not satisfy nodal point equilibrium, and additional iterations will be required. (Similar disturbance of equilibrium occurs after remeshing in an elastic-plastic updated Lagrange simulation.)

Therefore the integration point values are not completely replaced by the smoothed values obtained from equation (15), but are calculated as a weighed sum of equations (14) and (15). The weight factor $\alpha$ for global smoothing is proportional to the ratio of the relative displacement $\Delta \mathbf{u}-\Delta \mathbf{x}^{\mathrm{g}}$ and the element size, i.e. no global smoothing occurs if $\Delta \mathbf{u}=\Delta \mathbf{x}^{\mathbf{g}}$ (updated Lagrange).

$$
\begin{aligned}
\varepsilon\left(\mathbf{x}^{\mathrm{g}}+\Delta \mathbf{x}^{\mathrm{g}}, t+\Delta t\right)= & (1-\alpha) \varepsilon\left(\mathbf{x}^{\mathrm{g}}, t\right)+\alpha \psi^{N}\left(\mathbf{x}^{\mathrm{g}}\right) \varepsilon^{N}(t) \\
& +\Delta \varepsilon^{\mathrm{m}}+\left(\Delta \mathbf{x}^{\mathrm{g}}-\Delta \mathbf{u}\right) \cdot \vec{\nabla} \psi^{N} \varepsilon^{N}
\end{aligned}
$$

A reasonable range for $\alpha$ appeared to be

$$
\frac{\left|\Delta x^{\mathrm{g}}-\Delta u\right|}{l_{\mathrm{e}}} \leqslant \alpha \leqslant 2 \frac{\left|\Delta x^{\mathrm{g}}-\Delta u\right|}{l_{\mathrm{e}}}
$$

where $l_{\mathrm{e}}$ represents the element size.

The effect of the weighed global smoothing is shown by a simple one-dimensional strain propagation example. A finite element mesh with 20 elements is shown in Figure 1. Only one element in the middle of the mesh is subjected to simple shear, whereas all other elements are 


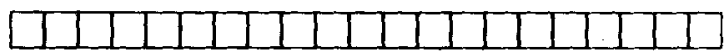

Figure 1. Finite element mesh to test prediction of convection of locally generated strain

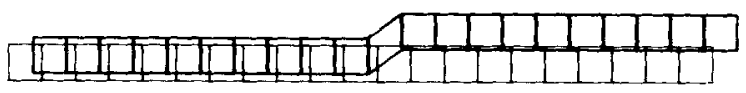

Figure 2. Distorted mesh showing the local straining due to an incremental material displacement

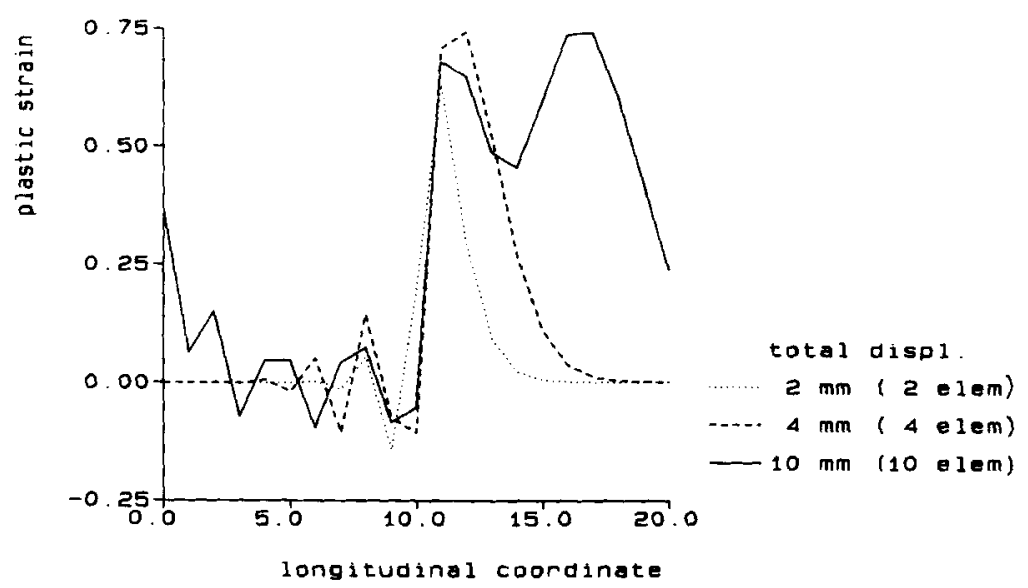

Figure 3. Predicted strain propagation in an Eulerian mesh without applying global smoothing

translating accordingly without deformation, as shown by a material displacement increment in Figure 2. The mesh is spatial fixed $\left(\Delta x^{8}=0\right)$.

This example can be regarded as numerical approximation of a slip line (local plastic deformation in an area with a thickness of one element). A simulation has been carried out with a coarse mesh ( 20 elements) and with a refined mesh (60 elements).

Figure 3 shows the predicted strain propagation without applying global smoothing. Figure 4 shows the predicted strain propagation with weighed global smoothing. The weight factor $\alpha$ is chosen in the middle of the range given by expression (17).

The total strain in the 'slip line' can analytically be calculated:

$$
\varepsilon=\frac{1}{\sqrt{3}}=0.57
$$

The predicted strain level in the simulation with weighed global smoothing shows a good agreement with the exact solution.

After the steady state is arrived at in the area around the local strain source, the prescribed incremental displacement field has been changed to pure longitudinal translation without local deformation. A propagation of the 'wave front' shown by Figure 5 is predicted which is in accordance with equation (8); if the material rate of change $\dot{\varepsilon}^{\mathrm{m}}$ vanishes, this expression reduces to a wave equation. The sharp edges are smoothed during the propagation resulting in a less steep front. A comparison of the results obtained with 20 elements and 60 elements shows that the numerical diffusion decreases when the mesh is refined, whereas no instabilities occur. 

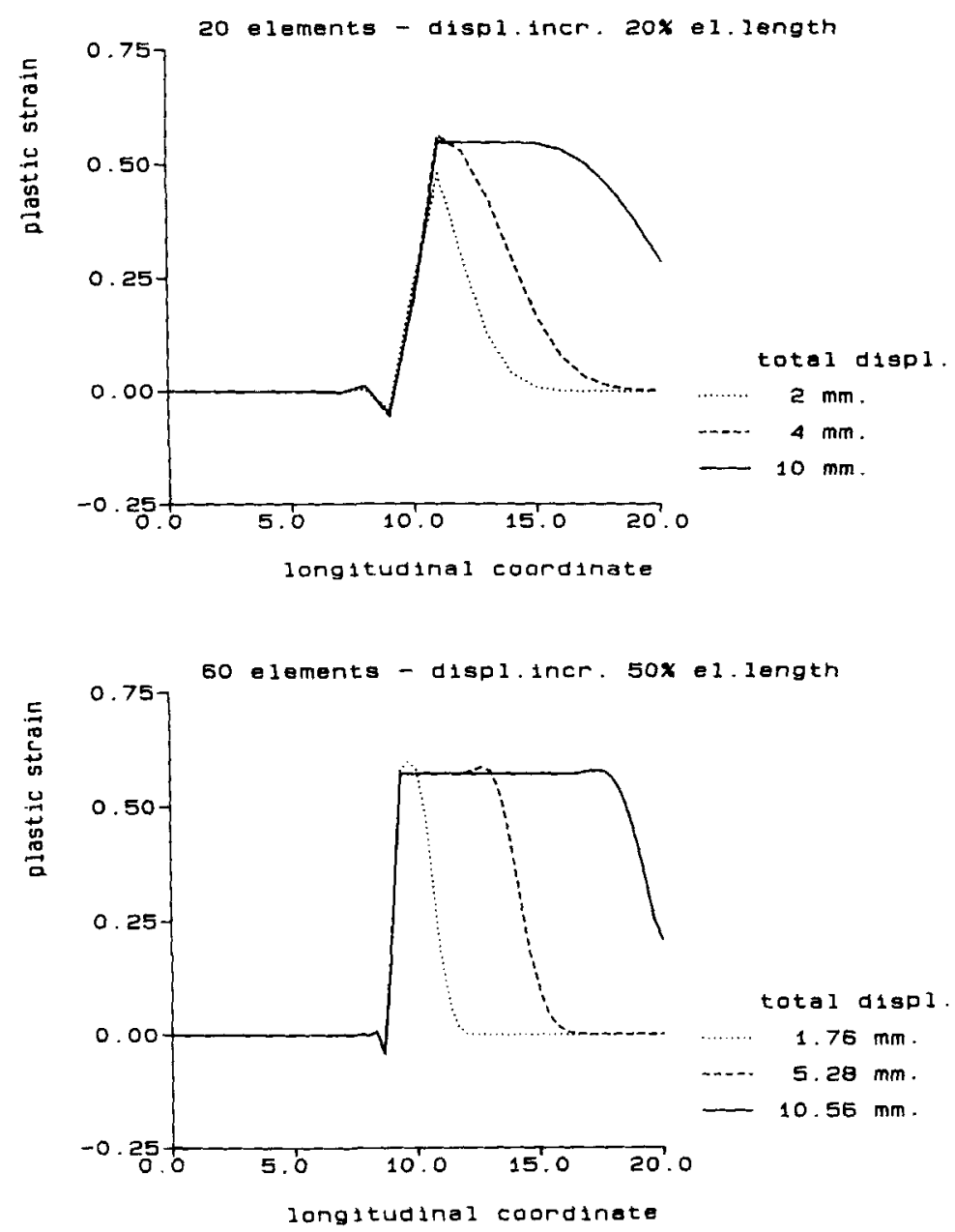

Figure 4. Predicted strain propagation with weighed global smoothing, with 20 elements and with 60 elements

Simulations have been carried with various step sizes and number of elements. The amount of smoothing appeared to be proportional to the element size, and to the total displacement, but is independent of the step size.

In the finite element program the weight factor for global smoothing is automatically taken into account at element level. To prevent numerical instabilities, the size of the relative displacement increments should be limited to less than half the element size.

For elements with quadratic or higher order of interpolation the accuracy of the procedure can be improved by considering that the first and last terms in the right hand side of equation (11) can be regarded as the first terms of a Taylor series expansion of the strain distribution. Hence equation (11) can be replaced by

$$
\varepsilon\left(\mathbf{x}^{\mathrm{g}}+\Delta \mathbf{x}^{\mathrm{g}}, t+\Delta t\right)=\varepsilon\left(\mathbf{x}^{\mathrm{g}}+\Delta \mathbf{x}^{\mathrm{g}}-\Delta \mathbf{u}, t\right)+\Delta \varepsilon^{\mathrm{m}}
$$

Consequently equation (16) can be replaced by

$$
\varepsilon\left(\mathbf{x}^{\mathrm{g}}+\Delta \mathbf{x}^{\mathrm{g}}, t+\Delta t\right)=(1-\alpha) \varepsilon\left(\mathbf{x}^{\mathrm{g}}, t\right)+\alpha \psi^{N}\left(\mathbf{x}^{\mathrm{g}}+\Delta \mathbf{x}^{\mathrm{g}}-\Delta \mathbf{u}\right) \varepsilon^{N}(t)+\Delta \varepsilon^{\mathrm{m}}
$$



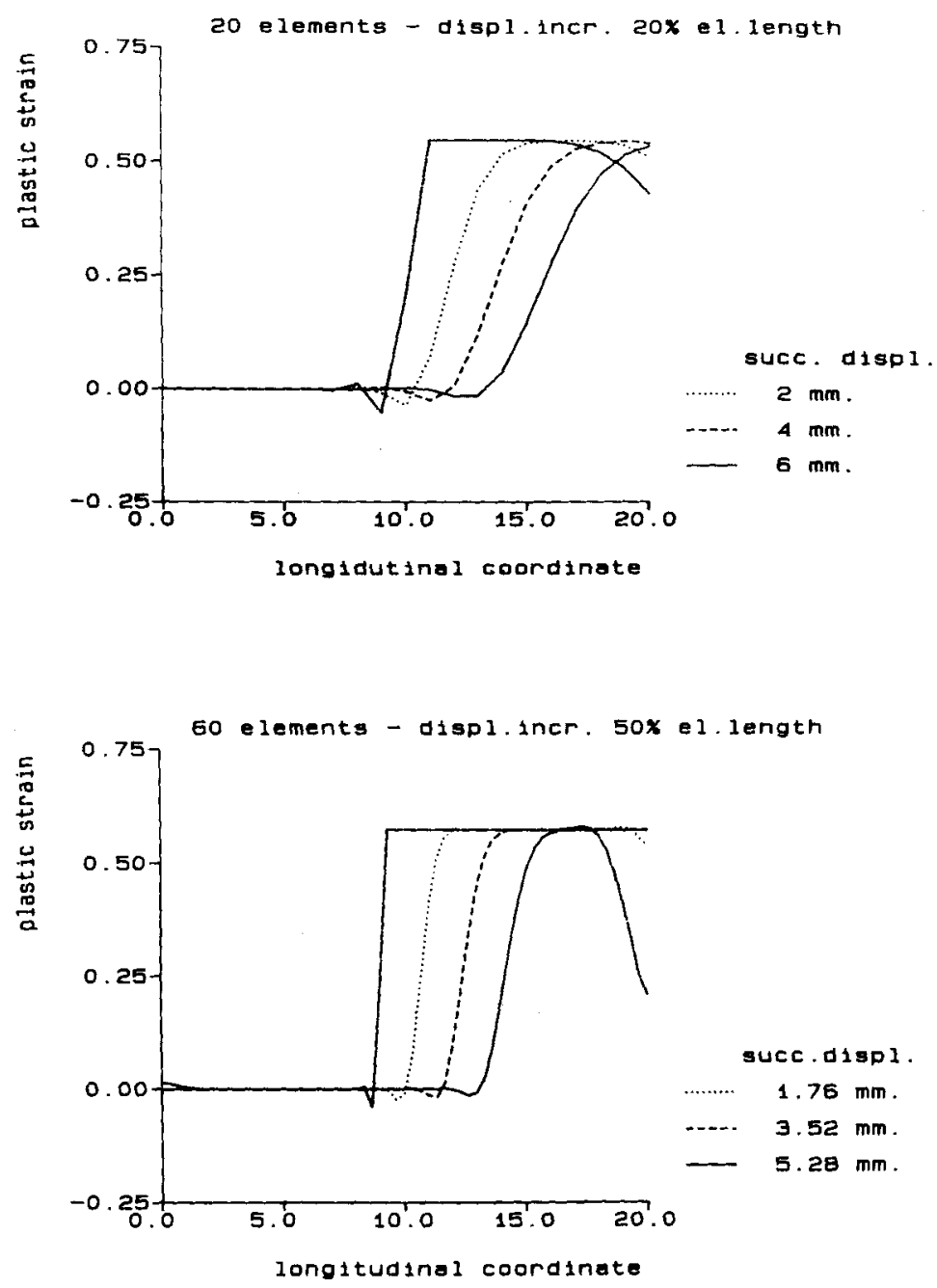

Figure 5. Predicted pure convection of plastic strain distribution, with 20 elements and 60 elements

The same procedure as shown here for the incremental calculation of the effective strain is also applied to stresses and other material associated quantities. The total displacement can be calculated in a similar way. Subtracting the total displacement from the final state must show a recovery of the initial shape. Deviations from the initial shape are a measure of the accuracy of the simulation.

Calculation of the mean nodal point value of strain and stress is restricted to those elements that represent the same kind of material because at material boundaries the stress components are not necessarily continuous. In contact elements, for example, the stress components are related to a local co-ordinate system defined by the orientation of the contact element. Consequently it is not possible to calculate nodal stresses as mean values of all adjacent elements. Therefore a procedure has been used by which the calculation of mean nodal stresses is restricted to elements of the same type. 


\section{PREDICTION OF STEADY-STATE, EULERIAN SIMULATION}

The first real process which is simulated is the wire drawing process. Neither thermal effect nor friction have been taken into account. The finite element mesh is shown in Figure 6. Isoparametric elements with four nodes and constant dilatation ${ }^{16}$ are used.

The reduction is accomplished by suppressing the velocity component normal to the contact surface between wire and die. The mixed Eulerian-Lagrangian method was applied. However, the spatial location of the outer surface hardly changes. Hence a purely Eulerian solution results. The simulation is carried out from the start up of the process until a stationary state is achieved. The stress-strain curve for the material is shown by Figure 7. Isotropic material satisfying the von Mises yield criterion is assumed.

Figure 8 shows the predicted equivalent plastic strain distribution for an increasing number of increments. It can be observed that the area in which a stationary state is achieved increases continuously. The diameter is reduced from 3.25 to $2.6 \mathrm{~mm}$. The corresponding homogeneous strain is then $2 \log (3 \cdot 25 / 2 \cdot 6)=0 \cdot 446$. This is indeed a lower bound of the predicted strain.

Figure 9 shows that the initial shape (i.e. diameter of the wire) is well recovered by subtracting the accumulated displacement.

The simulation of the wire drawing process shows that a steady-state solution can be found by a general non-steady-state solution method. Reduction of computer time may be possible if the special solution methods for a steady-state solution presented by Thompson and Szu-wei ${ }^{22}$ is used.

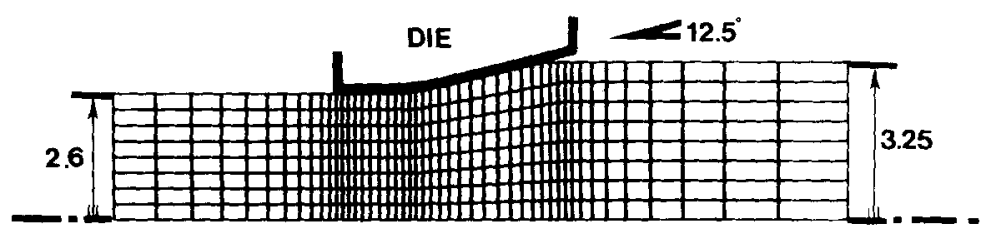

Figure 6. Finite element mesh for simulation of the wire drawing process

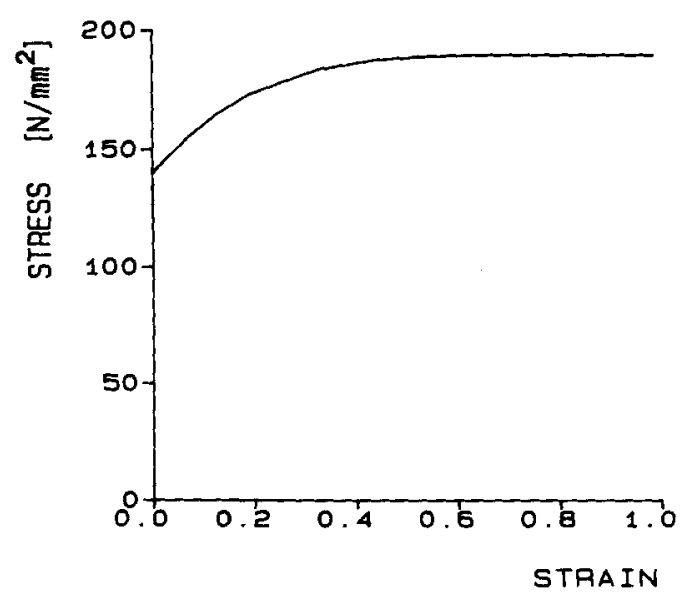

Figure 7. Stress-strain curve used in the simulation of the wire drawing process 

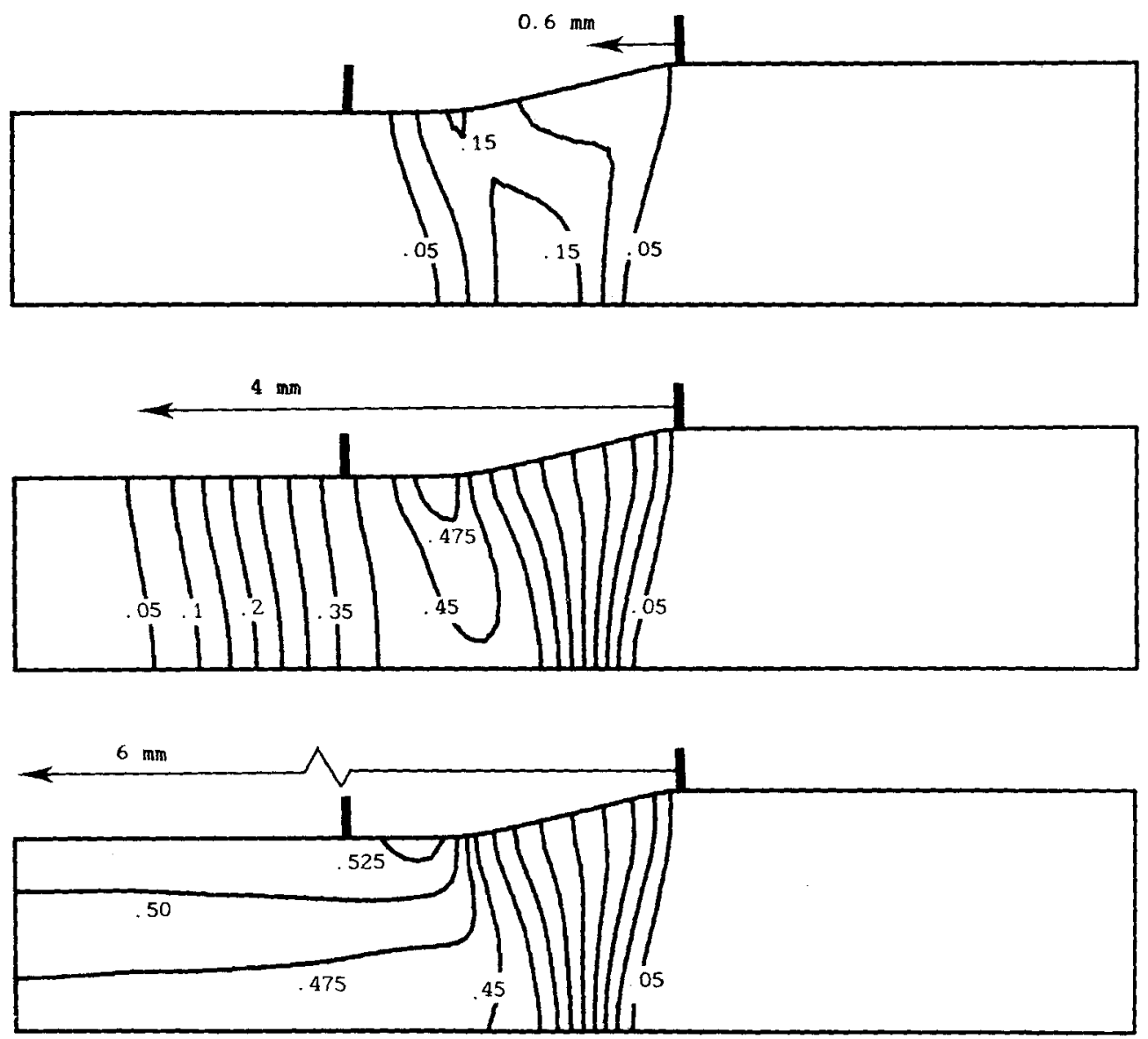

Figure 8. Predicted plastic strain propagation during the start up of the wire drawing process

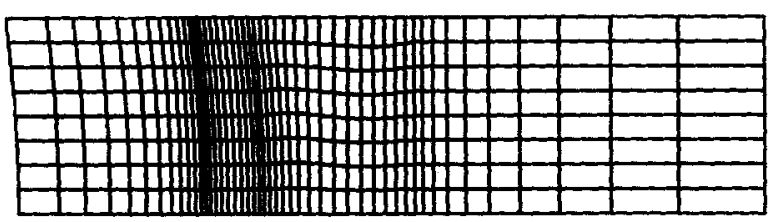

Figure 9. Recovered initial shape by subtracting the accumulated displacement from the actual nodal point co-ordinates

\section{CONTACT AND FRICTION}

A finite element formulation to model the contact conditions, especially in the multi-body case, has been presented by the authors ${ }^{10}$ in 1987. In the current paper the model is improved and extended to thermal aspects. An extensive formulation is given by Van der Lugt. ${ }^{13}$ The contact description is inspired on the assumption of a friction layer with a small but finite thickness. Stick-slip constitutive behaviour is introduced as non-associated elasto-plasticity. 
Consider two bodies A and B and a (candidate) contact region C. Define a reference surface $S_{\mathrm{C}}$ between the bodies, having equal normal or directional distances to either body. Neighbouring points at the two bodies and the intermediate reference surface are related by

$$
\begin{aligned}
& \mathbf{x}^{A}=\mathbf{x}^{\mathbf{C}}+\frac{1}{2} \Delta \mathbf{x} \\
& \mathbf{x}^{\mathbf{B}}=\mathbf{x}^{\mathrm{C}}-\frac{1}{2} \Delta \mathbf{x}
\end{aligned}
$$

Referring to Figure 10, two co-ordinate systems are defined: a global, fixed, co-ordinate system with orthonormal basis vectors $\left(\mathbf{b}_{1}, \mathbf{b}_{2},\left(\mathbf{b}_{3}\right)\right)$ and a local, co-rotating, co-ordinate system with local orthonormal basis vectors $\left(\mathbf{e}_{1}, \mathbf{e}_{2},\left(\mathbf{e}_{3}\right)\right)$ at the reference surface. The normal direction coincides with $e_{1}$, the tangential directions with $e_{2}$ and $e_{3}$ respectively. The orientation of the local basis not only depends on the location but changes also with time $\mathbf{e}_{i}=\mathbf{e}_{i}(\mathbf{x}, t)$. The rate of change of $\dot{\mathbf{e}}_{i}$ yields

$$
\dot{\mathbf{e}}_{i}=\frac{\partial \mathbf{e}_{i}}{\partial \mathbf{x}} \cdot \mathbf{v}+\frac{\partial \mathbf{e}_{i}}{\partial t}
$$

The first term at the right hand side of equation (21) represents the effect of the curvature of the reference surface and can formally be written using the Christoffel symbol as

$$
\frac{\partial \mathbf{e}_{i}}{\partial x_{k}} v_{k}=\frac{\partial^{2} \mathbf{x}}{\partial x_{i} \partial x_{k}} v_{k}=\mathbf{e}_{j} \Gamma_{j, i k} v_{k}=\mathbf{K}_{i} \cdot \mathbf{v}
$$

with $x_{k}=\mathbf{x} \cdot \mathbf{e}_{k}, v_{k}=\mathbf{v} \cdot \mathbf{e}_{k}$ and $\mathbf{K}_{i}=\mathbf{e}_{j} \Gamma_{j, i k} \mathbf{e}_{k}$; the curvature of the surface perpendicular to $\mathbf{e}_{i}$.

The second term of equation (21) accounts for the change of the shape including rigid rotation of the reference surface. The change of the shape and rotation can be related to the gradient of the normal component only. Formally we can write

with

$$
\frac{\partial \mathbf{e}_{i}}{\partial t}=\mathbf{e}_{i} \cdot \boldsymbol{\Omega}
$$

Here the normal component $\boldsymbol{9}^{\mathrm{n}}$ of the velocity can be expressed as

$$
\boldsymbol{\vartheta}^{\mathbf{n}}=\mathscr{N} \cdot \mathbf{v} \text { with }\left[\mathscr{N}_{i j}\right]=\left[\begin{array}{lll}
1 & 0 & 0 \\
0 & 0 & 0 \\
0 & 0 & 0
\end{array}\right]
$$

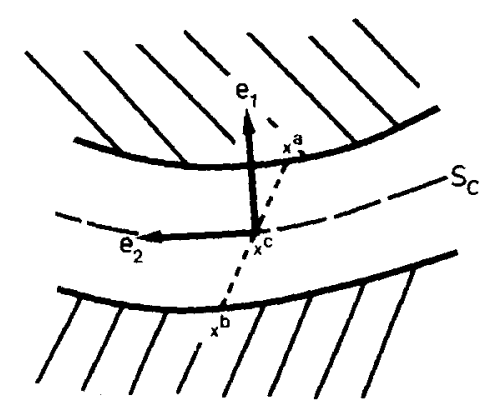

Figure 10. Position of related points in the contact area 
The spin tensor is skew-symmetric $\left(\Omega=-\Omega^{\mathrm{T}}\right)$. Consequently we can write

$$
\dot{\mathbf{e}}_{i}=\mathbf{K}_{i} \cdot \mathbf{v}+\mathbf{e}_{i} \cdot \mathbf{\Omega}=\mathbf{K}_{i} \cdot \mathbf{v}-\mathbf{\Omega} \cdot \mathbf{e}_{i}
$$

The rate of deformation $d_{i}$ in the contact area is defined as the rate of change of the local components of the (distance) vector between two neighbouring points on surface $S_{\mathrm{A}}$ and $S_{\mathrm{B}}$ respectively:

$$
d_{i}=\dot{x}_{i}^{\mathrm{A}}-\dot{x}_{i}^{\mathrm{B}}=\Delta \dot{x}_{i}
$$

Note that $v_{i}$ represents the components of absolute velocity whereas $\dot{x}_{i}$ is the relative velocity with respect to the local basis.

The vector $\mathbf{d}$ can be expressed as

$$
\mathbf{d}=d_{i} \mathbf{e}_{i}=\Delta \dot{x}_{i} \mathbf{e}_{i}=\frac{\mathrm{d}}{\mathrm{dt}}\left(\Delta x_{i} \mathbf{e}_{i}\right)-\Delta x_{i} \dot{\mathbf{e}}_{i}
$$

Substitution of (25) into (27) yields

$$
\mathbf{d}=\Delta \mathbf{v}+\Delta x_{i}\left(-\mathbf{K}_{i} \cdot \mathbf{v}+\mathbf{e}_{i} \cdot \mathbf{\Omega}\right)=\Delta \mathbf{v}+\Delta \mathbf{x} \cdot \mathbf{\Omega}^{\mathrm{C}}
$$

where

$$
\mathbf{\Omega}^{\mathrm{C}}=-\mathbf{e}_{i} \mathbf{K}_{i} \cdot \mathbf{v}+\mathbf{\Omega}
$$

The tensor $\mathbf{\Omega}^{\mathrm{C}}$ is skew-symmetric too, hence

$$
\mathbf{d}=\Delta \mathbf{v}-\mathbf{\Omega}^{\mathrm{C}} \cdot \Delta \mathbf{x}
$$

Evidently, the rate of deformation is found by correction of the difference between the surface velocities for rotation of the reference surface in a way similar to the co-rotational Jaumann rate. The rate of deformation equals zero in the case of rigid body rotation.

Note: If the local direction of $\Delta \mathbf{x}$ coincides with the normal direction $\left(\Delta x_{i}=0\right.$ for $\left.i=2,3\right)$ then there is only a contribution of the curvature of the reference surface. This contribution depends on the tangential components of the velocity only because the normal direction in the contact area can assumed to be straight $\left(\partial \mathbf{e}_{i} / \partial x_{1}=\mathbf{0}\right)$. The additional terms depending on the curvature are in this case similar to those found by Rebelo et al. ${ }^{19}$

Since contact stresses are considered to be tractions applying to only one reference surface $S_{\mathrm{C}}$, it can be denoted as a vector $\tau$ :

$$
\tau=\tau_{i} \mathbf{e}_{i}
$$

Taking time derivatives yields

$$
\dot{\tau}=\dot{\tau}_{i} \mathbf{e}_{i}+\tau_{i} \dot{\mathbf{e}}_{i} \Leftrightarrow \dot{\tau}=\dot{\tau}_{i} \mathbf{e}_{i}+\Omega_{i j}^{\mathrm{C}} \tau_{j} \mathbf{e}_{i}
$$

Alternatively $i$ can be subdivided into a co-rotational derivative $\dot{\tau}$ and a rotational change $\Omega^{\mathrm{C}} \cdot \tau$ :

$$
\dot{\tau}=\bar{\tau}+\Omega^{\mathrm{c}} \cdot \tau
$$

Hence, tensor components for $\stackrel{\nabla}{\tau}$ must satisfy

$$
\stackrel{\nabla}{\tau}=\dot{\tau}_{i} \mathbf{e}_{i}
$$

The rate of deformation is assumed to consist of a reversible (elastic) part and a non-reversible (plastic, i.e. slip) part. The normal relative displacement is strictly elastic. The non-elastic rate of deformation has the direction of the tangential stress (non-associated). The slip criterion used is Coulomb friction and $\mu$ denotes the friction coefficient. The constitutive contact behaviour results in

$$
\stackrel{\nabla}{\tau}=\mathbf{E} \cdot \mathbf{d}-\mathbf{Y} \cdot \mathbf{d}=\mathbf{C} \cdot \mathbf{d}
$$


where the components of $\mathbf{E}$ and $\mathbf{Y}$ for the two-dimensional case are

$$
[\mathbf{E}]=\left[\begin{array}{ll}
E_{11} & \phi \\
\phi & E_{22}
\end{array}\right] \text { and }[\mathbf{Y}]=\left[\begin{array}{cc}
\phi & \phi \\
\mu \operatorname{sgn}\left(\tau_{2}\right) E_{11} & E_{22}
\end{array}\right]
$$

The above relation is valid in the case of slip (plastic) deformation. For the elastic (stick) situation the yield tensor vanishes; $\mathbf{Y}=\phi$ : for the open situation the elastic tensor vanishes too; $\mathbf{C}=\mathbf{Y}=\mathbf{E}=\phi$.

\section{EXTENSIONS OF THE FINITE ELEMENT FORMULATION FOR CONTACT CONDITIONS}

For the two bodies A, B (Figure 10) the 'weak form' of the mechanical equilibrium equation (2) yields

$$
\int_{V_{\mathrm{A}}+V_{\mathrm{B}}} \delta \mathrm{d}: \sigma \mathrm{d} V-\int_{V} \delta \mathbf{v} \cdot \rho \mathbf{f} \mathrm{d} V+\int_{S_{\mathrm{c}}} \delta(\Delta \mathbf{v}) \cdot \tau \mathrm{d} S-\int_{S} \delta \mathbf{v} \cdot \mathbf{t} \mathrm{d} S=\phi \quad \forall \delta \mathbf{v}
$$

where the vector $t$ represents the surface traction per unit surface. $S_{\mathrm{C}}$ is the reference surface. The 'weak form' of the thermal equilibrium equation (3) results in

$$
\begin{aligned}
& \int_{V_{\mathrm{A}}+V_{\mathrm{B}}}(\delta \dot{T} \rho c \dot{T}+\vec{\nabla} \delta \dot{T} \cdot \lambda \cdot \vec{\nabla} T-\delta \dot{T} \boldsymbol{\sigma}: \mathbf{d}) \mathrm{d} V+\int_{S} \delta \dot{T} \boldsymbol{\varphi} \cdot \mathbf{n} \mathrm{d} S \\
& \quad+\int_{S_{\mathrm{c}}}\|\Delta \mathbf{x}\|(\delta \dot{T} \rho c \dot{T}+\overrightarrow{\mathbf{v}} \delta \dot{T} \cdot \lambda \cdot \vec{\nabla} T-\delta \dot{T} \tau \cdot \mathbf{d}) \mathrm{d} S=\phi \quad \forall \delta \dot{T}
\end{aligned}
$$

The description of the contact element is derived from the rate of change of the contact surface integral in (16).

$$
\frac{\mathrm{d}}{\mathrm{d} t} \int_{S_{\mathrm{c}}} \delta(\Delta \mathbf{v}) \cdot \tau \mathrm{d} S=\int_{S_{\mathrm{c}}}(\delta \Delta \mathbf{v} \cdot \mathbf{C} \cdot \mathbf{d}+\delta \Delta \mathbf{v} \cdot \boldsymbol{\Omega} \cdot \tau) \mathrm{d} S+\int_{\mathrm{S}_{\mathrm{c}}}\left(\delta \Delta \mathbf{v} \cdot \tau \vec{\nabla}_{\mathrm{s}} \cdot \mathbf{v}_{\mathrm{s}}\right) \mathrm{d} S
$$

The real velocity distribution and temperature are approximated by interpolation of nodal point velocities $\mathbf{v}^{N}$ and nodal point temperatures $T^{N}$. The contact layer has a finite thickness, therefore the contact elements are formulated as bulk elements with linear interpolation in thickness direction. This approximation yields

$$
\begin{gathered}
\mathbf{v}=\sum_{N} \psi^{N} \mathbf{v}^{N}, \quad T=\sum_{N} \psi^{N} T^{N} \\
\Delta \mathbf{v}=\sum_{N} \mathbf{B}^{N} \cdot \mathbf{v}^{N}, \quad \mathbf{d}=\sum_{N} \mathbf{D}^{N} \cdot \mathbf{v}^{N} \quad \text { and } \quad \mathbf{\Omega}^{\mathrm{C}}=\sum_{M} \mathbf{W}^{M} \cdot \mathbf{v}^{M}
\end{gathered}
$$

where

$$
\begin{aligned}
\mathbf{B}^{N} & =\left(\overrightarrow{\mathbf{\nabla}} \psi^{N} \cdot \Delta \mathbf{x}\right) \mathbf{I}=\left(\mathbf{B}^{N}\right)^{\mathrm{T}} \\
\mathbf{D}^{N} & =\left(\vec{\nabla} \psi^{N} \cdot \Delta \mathbf{x}\right) \mathbf{I}-\Delta x_{i} \mathbf{K}_{i} \psi^{N}-\left(\left(\vec{\nabla} \psi^{N} \cdot \Delta \mathbf{x}\right) \mathcal{N}-\vec{\nabla} \psi^{N} \Delta \mathbf{x} \cdot \mathcal{N}\right) \\
\mathbf{W}^{N} & =-\mathbf{e}_{i} \mathbf{K}_{i} \psi^{N}+\left(\mathbb{L}:\left(\overrightarrow{\mathbf{\nabla}} \psi^{N} \cdot \mathcal{N}\right)-\vec{\nabla} \psi^{N} \cdot \mathcal{N}\right)
\end{aligned}
$$

The fourth order tensor $\mathbb{L}$ is the 'transposed identity' tensor $\mathbb{L}: \mathbf{X}=\mathbf{X}^{\mathbf{T}}$ for any $\mathbf{X}$. The component of $\mathbb{L}$ are $\mathbb{L}_{i j k l}=\delta_{j k} \delta_{i l}$.

For assembling finite elements the nodal point velocities have to be expressed in components referring to the global directions. The transformation from the global to the local basis is represented by

$$
\mathbf{e}_{i}=R_{i x} \mathbf{b}_{\alpha}
$$


where $R_{i \alpha}$ are the components of an orthonormal matrix. Components of vectors and tensors referring to the global directions are indicated by Greek subscripts, components referring to the local directions are indicated by Latin lower case subscripts.

The relation between local and global velocity components is then

$$
v_{i}=R_{i \alpha} v_{\alpha}
$$

Components of $\mathbf{D}^{N}$ and $\mathbf{W}^{N}$ including transformation to a local co-ordinate system can be written as

$$
\begin{aligned}
D_{i \alpha}^{N} & =\frac{\partial \psi^{N}}{\partial x_{m}} \Delta x_{m} R_{i \alpha}-\psi^{N} \Delta x_{m} \Gamma_{i, m j} R_{j \alpha}-\left(\frac{\partial \psi^{N}}{\partial x_{m}} \Delta x_{m} \mathcal{N}_{i j} R_{j \alpha}-\frac{\partial \psi^{N}}{\partial x_{i}} \Delta x_{m} \mathcal{N}_{m j} R_{j \alpha}\right) \\
W_{i j \alpha}^{N} & =-\psi^{N} \Gamma_{j, i m} R_{m \alpha}+\left(\frac{\partial \psi^{N}}{\partial x_{j}} \mathcal{N}_{i m} R_{m \alpha}-\frac{\partial \psi^{N}}{\partial x_{i}} \mathcal{N}_{j m} R_{m \alpha}\right)
\end{aligned}
$$

Note: The interpolation functions $\psi^{N}$ are linear in the thickness direction, hence the factor $\left(\partial \psi^{N} / \partial x\right) \Delta x$ is approximately equal to one and does not vanish when contact $(\Delta x \rightarrow 0)$ occurs.

Substitution of (39) and (40) into the contact surface integrals of (37) and (38) results into contact/slip matrices with components

$$
\begin{aligned}
K^{M N} & =\int_{S_{\mathrm{c}}}\left(\mathbf{B}^{M} \cdot \mathbf{C} \cdot \mathbf{D}^{N}+\mathbf{B}^{M} \cdot \tau: \mathbf{W}^{N}\right) \mathrm{d} S, & & K_{\mathrm{s}}^{M N}=\int_{S_{\mathrm{c}}} \mathbf{B}^{M} \cdot \tau \nabla \psi^{N} \mathrm{~d} S \\
K_{\mathrm{c}}^{M N} & =\int_{S_{\mathrm{c}}}\|\Delta \mathbf{x}\| \psi^{M} \rho c \psi^{N} \mathrm{~d} S, & & K_{q}^{M N}=\int_{S_{\mathrm{c}}} \psi^{N} \tau \cdot \mathbf{D}^{N} \mathrm{~d} S \\
K_{\lambda}^{M N} & =\int_{S_{\mathrm{c}}} \vec{\nabla} \psi^{N} \cdot \lambda_{\mathrm{s}} \cdot \vec{\nabla} \psi^{N} \mathrm{~d} S & &
\end{aligned}
$$

We can regard all nodal degrees of freedom as an ordered collection, representing a multidimensional vector $[\dot{\mathbf{u}}]$. The sub vector with all velocity components is denoted by $[\mathbf{v}]$ and the sub vector with all temperature rates is denoted by $[\dot{\mathbf{T}}]$ :

$$
[\dot{\mathbf{i}}]^{\mathrm{T}}=\left[[\mathbf{v}]^{\mathrm{T}},[\dot{\mathbf{T}}]^{\mathrm{T}}\right]=\left[\left\{v_{\alpha}^{N}\right\},\left\{\dot{T}^{N}\right\}\right]
$$

The assembly of contact elements and body elements results in a set of linear algebraic equations represented by

$$
\left[\begin{array}{cc}
{\left[\mathbf{K}+\mathbf{K}_{s}\right]} & {\left[\mathbf{K}_{T}\right]} \\
{\left[\mathbf{K}_{q}\right]} & {\left[\mathbf{K}_{c}\right]}
\end{array}\right]\left[\begin{array}{l}
{[\mathbf{v}]} \\
{[\dot{\mathbf{T}}]}
\end{array}\right]=\left[\begin{array}{c}
{[\dot{\mathbf{F}}]} \\
-\left[\mathbf{K}_{\lambda}\right][\mathbf{T}]-\left[\mathbf{K}_{\mathbf{\Phi}}\right]
\end{array}\right]
$$

Note: The matrices $\left[\mathbf{K}_{T}\right]$ and $\left[\mathbf{K}_{\Phi}\right]$ contain contributions of the bulk elements only. They represent temperature dependent material properties and thermal boundary conditions respectively. A matrix $\left[\mathbf{K}_{T}\right]$ may result from a contact/slip element if slip conditions are temperature dependent.

\section{NON-STEADY-STATE PROBLEMS}

In order to assure that the nodal points on a moving (contact) surface remain on that surface (not necessarily at the same material particle), a procedure as illustrated in Figure 11 is followed. The material boundary is found after temporary updating of the nodal point co-ordinates with the material displacement increment. A spline is used to establish the material surface locally. The 
new nodal point location is found by the intersection of the spline with a line through the initial nodal point location in a predetermined direction (usually the normal direction).

It can be observed that the new locations of these points do not coincide with the surface that is obtained if one follows the element boundaries. However, if one chooses the new position of the nodal points on the surface obtained by using a spline through the material surface points, the amount of lost material is more or less compensated by the added material.

\section{Simulation of an upsetting process}

A billet is deformed by a punch, the initial shape being the same as used by Doltsinis and co-workers. ${ }^{1,2}$ The element mesh, shown in Figure 12, consists of 50 isoparametric elements with 4 nodes with constant dilatation ${ }^{15,16}$ and 14 contact elements. Figure 11 shows the distorted grid using the updated Lagrange method after 47 per cent height reduction. Some difficulties can be

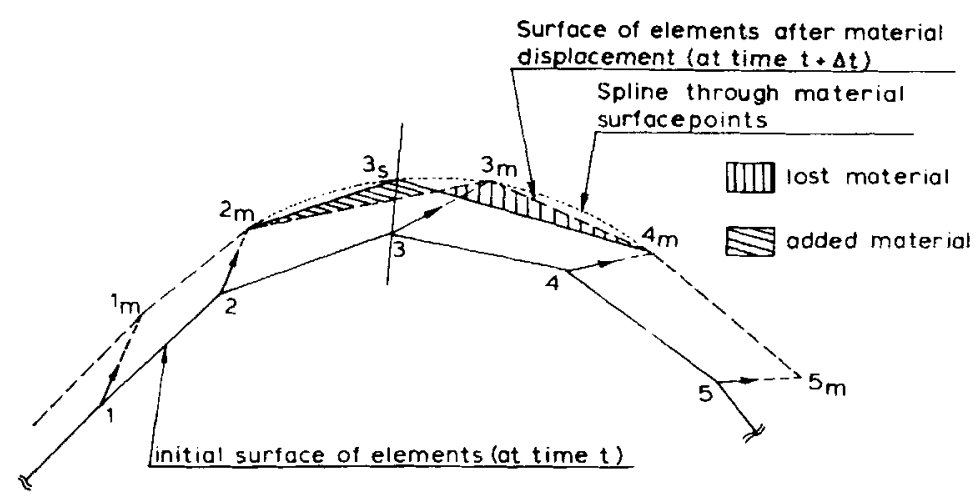

Figure 11. Adaptation of nodal surface point location

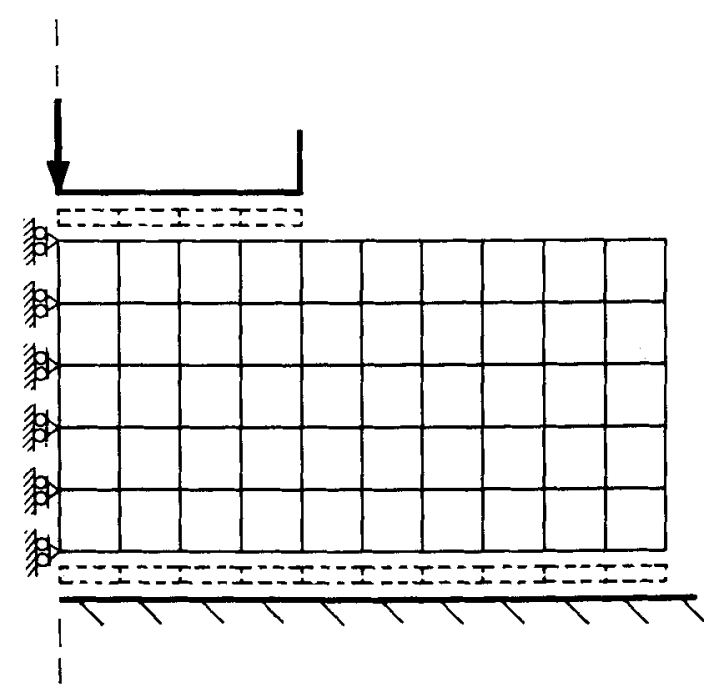

Figure 12. Element mesh of the upsetting simulation 


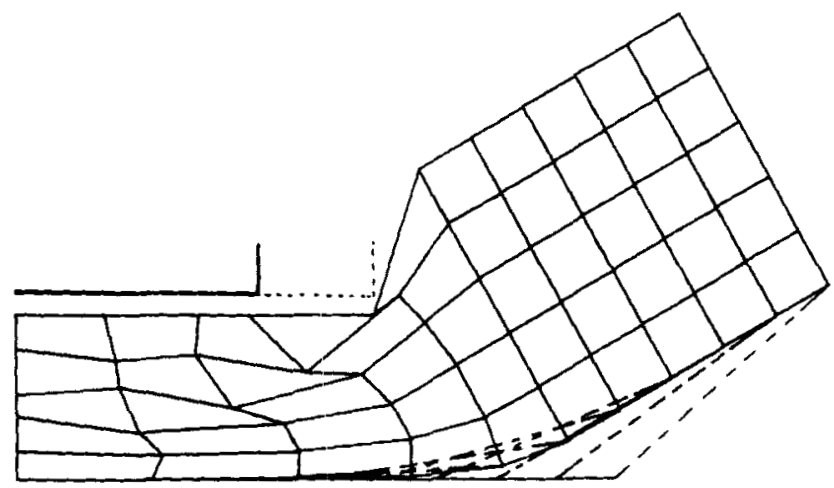

Figure 13. Distorted grid using updated Lagrange metbod

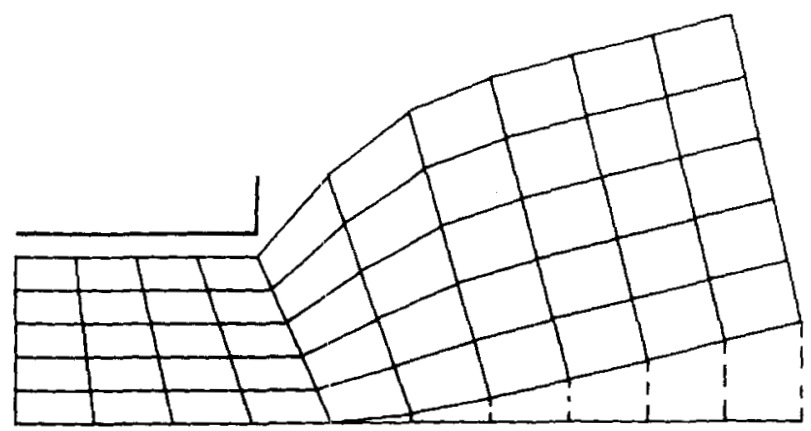

Figure 14. Deformed mesh using the mixed Eulerian-Lagrangian method

noted:

(i) the size of the punch increases apparently (Figure 13); change of geometry requires a special treatment of nodal boundary conditions;

(ii) the element mesh distorts owing to large deformations. Some elements are turned inside out, leading to numerical problems.

In contrast with the updated Lagrange method the present method does not suffer from this type of problem and can easily be continued, see Figure 14 . This can be considered as a real advantage of the mixed Eulerian-Lagrangian method.

The mesh used in this example was rather coarse. Therefore a simulation with a more refined mesh is carried out. The undeformed and the deformed mesh are shown by Figures 15 and 16 respectively. By subtracting the calculated total nodal point displacements from the final shape, the initial shape is recovered approximately, see Figure 17. It can be observed that a significant deviation from the initial shape is found near the edge of the punch. Local mesh refinement will probably reduce the deviation.

The method has also successfully been applied to simulations of cold rolling, ${ }^{13}$ deep drawing, forging and other forming processes. ${ }^{5,10,11,14,17,18}$ Similar procedures coupled with adaptive remeshing are used by Doltsinis, ${ }^{1,2}$ Huang et al. ${ }^{7}$ and Zienkiewicz and Huang. ${ }^{23}$ 


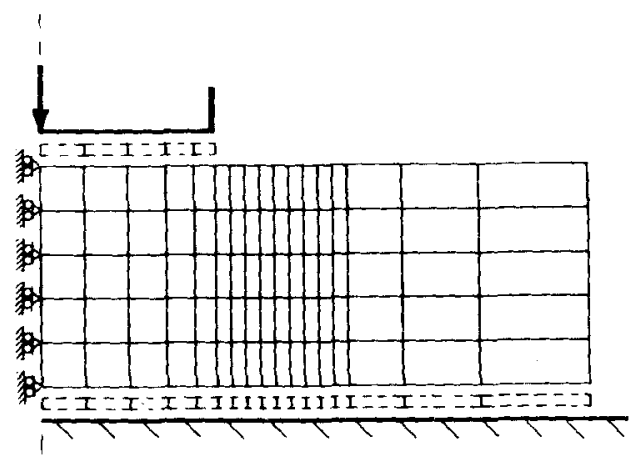

Figure 15. Undeformed refined mesh

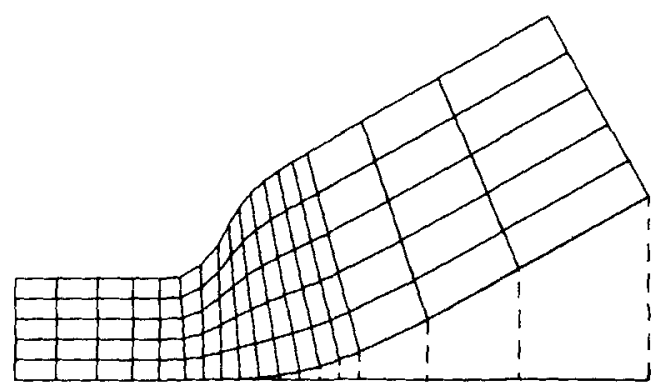

Figure 16. Deformed refined mesh

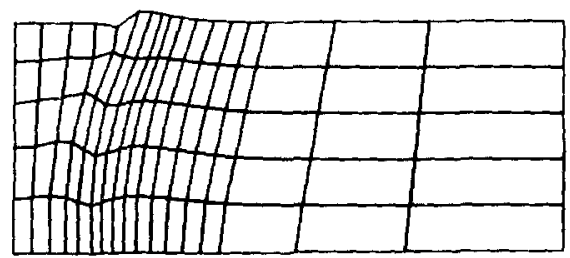

Figure 17. Recovered initial shape by subtracting the total displacement from the final shape

\section{CONCLUDING REMARKS}

The mixed Eulerian-Lagrangian finite element method (decoupling of material and grid displacement) is a powerful tool in the simulation of forming processes. Local and global smoothing is required to avoid numerical instabilities. Global smoothing results in numerical diffusion and disturbance of equilibrium. An optimum result is obtained by applying a weight factor for the amount of global smoothing which is proportional to the ratio of the displacement increment and the element size. The method can be regarded as complementary to remeshing.

\section{ACKNOWLEDGEMENTS}

The authors thank J. Brinkman and $M$. Louwes for carrying out the various numerical simulations and for their contribution to the presentation of the results. 


\section{REFERENCES}

1. J. St. Doltsinis and J. Luginsland, 'Unformspezifische Rechenferfahren Kontakt und Reibung, variabele Diskretisierung', Workshop Numerische Methoden der Plastomechanik, Hannover FRG (1986).

2. J. St. Doltsinis, J. Luginsland and S. Nolting, 'Some developments in the numerical simulation of metal forming processes', Int. Conf. on Computational Plasticity, Barcelona, Spain; Also Int. J. Eng. Computat., 4, 266-280 (1987).

3. J. Donea, 'Arbitrary Lagrangian-Eulerian finite element method', in T. Belytschko and T. J. R. Hughes (eds.), Computer Methods in Mechanics: Vol. 1. Computer Methods for Transient Analysis, North-Holland, Amsterdam, 1983.

4. C. J. M. Gelten and A. W. A. Konter, 'Application of mesh-rezoning in the updated Lagrangian method to metal forming analysis', in J. F. T. Pittman et al. (eds.), Numerical Methods in Industrial Forming Processes, Pineridge Press, Swansea, U.K., 1982, pp. 511-521.

5. S. Gosh, 'A new finite element description for simulation of metal forming processes', in E. G. Thompson et al. (eds.), Numerical Methods in Industrial Forming Processes, Balkema, Rotterdam, 1989, pp. 159-164.

6. E. Hinton and J. S. Gampbell, 'Local and global smoothing of discontinuous finite element functions using least square method', Int. j. numer. methods eng., 8, 461-480 (1974).

7. G. C. Huang, Y. C. Liu and O. C. Zienkiewicz, Error control, mesh updating schemes and automatic adaptive remeshing for finite element analysis of unsteady extrusion processes', in J. L. Chenot and E. Oñate (eds.), Modelling of Forming Processes, Kluwer Academic Publishers, Dordrecht, Netherlands, 1988, pp. 75-83.

8. J. Huétink, 'Analysis of metal forming processes based on a combined Eulerian-Lagrangian finite element formulation', in J. F. T. Pittman et al. (eds.), Numerical Methods in Industrial Forming Processes, Pineridge Press, Swansea, U.K., 1982, pp. 501-509.

9. J. Huétink, 'On the simulation of thermo-mechanical forming processes', Dissertation, University of Twente, The Netherlands, 1986.

10. J. Huétink, J. van der Lugt and J. R. Miedema, 'A mixed Euler-Lagrangian contact element to describe boundary and interface behaviour in forming processes', Proc. NUMETA, Vol. I, Nijhof, The Netherlands, 1987.

11. J. Huétink, P. T. Vreede and J. van der Lugt, 'The simulation of contact problems in forming processes using a mixed Euler-Lagrangian finite element method', in E. G. Thompson et al. (eds.), Numerical Methods in Industrial Forming Processes. Balkema, Rotterdam, 1989, pp. 549-554.

12. T. J. R. Hughes, W. K. Liu and T. K. Zimmermann, 'Lagrangian-Eulerian finite element formulation for incompressible viscous flow', Comp. Methods Appl. Mech. Eng., 29, 329-349 (1981).

13. J. van der Lugt, 'A finite element method for the simulation of thermo-mechanical contact problems in forming processes', Dissertation, University of Twente, The Netherlands, 1988.

14. J. van der Lugt and J. Huétink, 'Thermal-mechanially coupled finite element analysis in metal forming processes', Comp. Methods Appl. Mech. Eng., 54, 145-160 (1986).

15. J. C. Nagtegaal and J. E. de Jong, 'Some computational aspects of elastic-plastic large strain analysis', Int. j. numer. methods eng., 17, 15-41 (1981).

16. J. C. Nagtegaal, D. M. Parks and J. C. Rice, 'On numerically accurate finite element solutions in the fully plastic range', Comp. Methods Appl. Mech. Eng., 4, 153-177 (1974).

17. J. P. Ponthon, 'A method to reduce cost of mesh deformation in Eulerian-Lagrangian formulation', in J. L. Chenot and E. Oñate (eds.), Modelling of Forming Processe's, Kluwer Academic Publishers, 1988, pp. 65-74.

18. J. P. Ponthon, 'Efficient mesh management in Eulerian-Lagrangian method for large deformation analysis', in E. G. Thompson et al. (eds.), Numerical Methods in Industrial Forming Processes, Balkema, Rotterdam, 1989, pp. $203-210$.

19. N. Rebelo, J. C. Nagtegaal and H. D. Hibbitt, 'Practical aspects of modeling sheet forming processes', in E. G. Thompson et al. (eds.), Numerical Methods in Industrial Forming Processes, Balkema, Rotterdam, 1989, pp. 31-43.

20. P. J. G. Schreurs, 'Numerical simulation of forming processes', Dissertation, University of Eindhoven, The Netherlands, 1983.

21. P. J. G. Schreurs, F. E. Veldpaus and W. A. M. Brekelmans, 'An arbitrary Eulerian-Lagrangian finite element model for the simulation of geometrical non-linear hyper elastic and elasto-plastic deformation processes', in J. F. T. Pittman et al. (eds.), Numerical Methods in Industrial Forming Processes, Pineridge Press, Swansea, U.K., 1982, pp. 501-509.

22. E. G. Thompson and Yu Szu-wei, 'A direct Eulerian finite element method for steady state elastic plastic flow', in E. G. Thompson et al. (eds.), Numerical Methods in Industrial Forming Processes, Balkema, Rotterdam, 1989, pp. 95-103.

23. O. C. Zienkiewicz and G. C. Huang, 'Adaptive modelling of transient coupled metal forming processes', in E. G. Thompson et al. (eds.), Numerical Methods in Industrial Forming Processes, Balkema, Rotterdam, 1989, pp. 3-10.

24. O. C. Zienkiewicz and J. Z. Zhu, 'A simple error estimator and adaptive procedure for practical engineering analysis', Int. $j$. numer. methods eng., 24, 337-357 (1987). 